\title{
Factores de diferenciación génica y su futuro en el tratamiento de la osteoporosis: de la adipogénesis a la osteoblastogénesis, ¿del mismo modo y en sentido
} contrario?

\author{
Factors of gene differentiation and its future in the treatment of \\ osteoporosis: From adipogénesis to osteoblatogenesis, in the same way \\ and in the opposite direction?
}

\section{Pedro Sánchez Márquez¹, Carlos Arturo Révérend Lizcano²}

\author{
${ }^{1}$ Médico especialista en medicina interna. Fellow Endocrinología $2^{\circ}$ \\ año. Fundación Universitaria de Ciencias de la Salud. \\ ${ }^{2}$ Profesor Asistente, Facultad de Medicina. Fundación Universitaria \\ de Ciencias de la Salud-FUCS \\ Autor correspondencia: Pedro Sánchez Márquez. \\ pedros0822@hotmail.com. \\ Fecha de recepción: 8/04/2017 \\ Fecha de aceptación: 29/08/2018
}

\section{Resumen}

$\mathrm{E}$ l presente artículo tiene como objetivo presentar de forma resumida los diferentes factores que están involucrados en la diferenciación y el mantenimiento del fenotipo óseo, en contraste con los factores adipogénicos, cuya expresión determina procesos de diferenciación mutuamente excluyentes. Por otro lado, se propone el posible uso terapéutico para distintas patologías óseas como la osteoporosis. Los datos fueron obtenidos de estudios clínicos aleatorizados y de revisión, en idioma español e inglés, de los últimos 15 años, que incluyeran los términos Mesh: Osteoporosis; Osteoporoses; Osteoporosis, Post-Traumatic; Osteoporosis, Senile; Osteoporosis, Age-Related; Bone Loss, Age-Related; Factors, Transcription; Transcription Factor; Adipogeneses; Bone Formation; Osteoclastogenesis; Endochondral Ossification; Endochondral Ossifications; Ossification, Endochondral; Ossification, Physiological; Ossification, Physiologic.

Palabras clave: Células madre mesenquimales, osteoporosis, factores de transcripción, adipogénesis, osteoblastogénesis, Runx2, PPAR $\gamma$, BMP, Wnt/ B catenina, FGF, sLZIP, quemerinas/CMKLR1, miR223, miR30.

\section{Abstract}

The aim of this review is to summarize the different known factors involved in the differentiation and maintenance of the bone phenotype, in contrast to the adipogenic factors, whose expression determines mutually exclusive differentiation process. On the other hand, the possible therapeutic use for different bone diseases such as osteoporosis. The data was obtained from randomized clinical trials and review articles, in Spanish and English, from the last 15 years, which included the terms Mesh: Osteoporosis; Osteoporoses; Osteoporosis, Post-Traumatic; Osteoporosis, Senile; Osteoporosis, Age-Related; Bone Loss, Age-Related; Factors, Transcription; Transcription Factor; Adipogeneses; Bone Formation; Osteoclastogenesis; Endochondral Ossification; Endochondral Ossifications; Ossification, Endochondral; Ossification, Physiological; Ossification, Physiologic.

Keywords: Mesenchymal stem cell; Osteoporosis; Transcription factor; Adipogeneses; Osteoblastogénesis; Runx2; PPARY; BMP; Wht/ B catenin; FGF; sLZIP; Chemerin/CMKLR1; miR223; miR30.

La osteoporosis (OP) es resultado de una alteración esquelética caracterizada por el compromiso de la resistencia ósea, reflejada por cambios en la integración entre la densidad y la calidad del hueso, predispone a un cambio en la rigidez y menor flexibilidad, con el consecuente aumento del riesgo de fractura ${ }^{(1)}$. Según sus factores precipitantes, puede ser primaria o secundaria, la primera, corresponde al $80 \%$ de los casos en las mujeres y al $60 \%$ de los hombres, su origen es multifactorial y combina factores nutricionales, endocrinos, genéticos y mecánicos ${ }^{(2)}$ que resultan en el desacoplamiento de la unidad de remodelación ósea ${ }^{(3)}$, llevando a estimulación de la osteoclastogenia, con pérdida de la osteoblastogénesis y 
aumento proporcional de la adipogénesis ${ }^{(4)}$, y cuando se logra un desbalance entre la resorción y formación se presenta pérdida de la integridad estructural ${ }^{(5,6)}$. Estas alteraciones osteo/ adipogénicas se han observado en otras condiciones de pér-

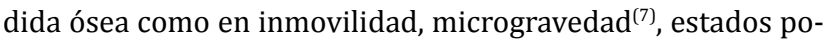
sooforectomía, diabetes ${ }^{(8)}$ y con el uso de medicamentos como esteroides $^{(9)}$.

En la médula ósea coexisten múltiples tipos de células, de las cuales hasta el $0,01 \%$ corresponde a células madre mesenquimales (MSC) con capacidad de diferenciación multilinaje, incluyendo varias líneas celulares mesodérmicas como la osteoblástica, condrocítica, miocítica y adipocítica, así como líneas no mesodérmicas como el músculo cardiaco y la piel ${ }^{(10,11)}$ (figura 1). El direccionamiento hacia cada línea celular está influenciado por factores estimulantes e inhibidores que generan una relación reciproca e inversa por diferenciación competitiva; su presencia puede, incluso, lograr desdiferenciación y reprogramación celular.

Entre las células especializadas se encuentran los osteoblastos, los cuales permiten el desarrollo del esqueleto óseo a través de osificación condral y acondral, para lo cual requieren de la expresión secuencial y sostenida de factores de transcripción que estimulan su diferenciación y mantenimiento del fenotipo, esto se logra por la acción de moléculas señalizadoras de tipo ligando que al interactuar con su receptor activan diversos mecanismos de transducción de señal.

Dentro de los factores osteoblastogénicos más importantes se encuentran el factor de transcripción asociado a Runt (Runx2), la osterexina (Osx) y el Dlx5 $5^{(12)}$, su fenotipo final se evidencia por la expresión de marcadores tempranos y tardíos de diferenciación como el colágeno tipo 1 (Col1), la proteína secretora rica en ácido cisteínico (SPARC), la osteopontina (Opn), la sialoproteína ósea (BSP), la osteocalcina (Osc) y la fosfata- sa alcalina (ALP). Dentro de las moléculas señalizadoras que direccionan la diferenciación se encuentran las proteínas morfogénicas óseas (BMP), la Wnt/B catenina, los factores de crecimiento fibroblástico (FGF), en tanto que los microARN (miARN), proteínas pequeñas de leucina (sLZIP) y las quemerinas actúan como moduladores de la expresión.

En el caso de la adipogénesis, el factor de transcripción más importante es el PPAR $\gamma^{(13,14,15)}$, el cual se activa por moléculas de señalización tipo proteínas de unión al activador de CAAT (CCAAT) y los potenciadores de unión a proteínas (EBP), donde los tipos $\beta$ y $\gamma$ se encargan de la diferenciación temprana mientras el $\alpha$ se encarga de la tardía. Su fenotipo se asocia a la expresión de marcadores tipo lipoproteína lipasa (LPL) y apoproteína a2 (aP2) (figura 2).

Este artículo de revisión tiene como objetivo presentar de forma resumida los diferentes factores que están involucrados en la diferenciación y el mantenimiento del fenotipo óseo, en contraste con los factores adipogénicos, cuya expresión determina procesos de diferenciación mutuamente excluyentes. Por otro lado, se propone el posible uso terapéutico para distintas patologías óseas como la osteoporosis.

\section{BMPs}

Clasificados dentro de la superfamilia del factor de crecimiento transformante beta (TGF- $\beta$ ), en humanos, han sido descritos 15 miembros de esta familia de proteínas. Con expresión en distintos tejidos, incluyendo riñón, ojos, cartílago y tejido reproductivo, tienen un papel importante en la embriogénesis, organogénesis y proliferación celular, a nivel óseo tienen habilidad osteoinductiva, descrita inicialmente en huesos desmineralizados $^{(10)}$.

$\mathrm{Su}$ actividad señalizadora inicia cuando el BMP se une al complejo heterodimérico de receptor transmembrana serina/

Figura 1. Diferenciación de las células mesenquimales multipotenciales

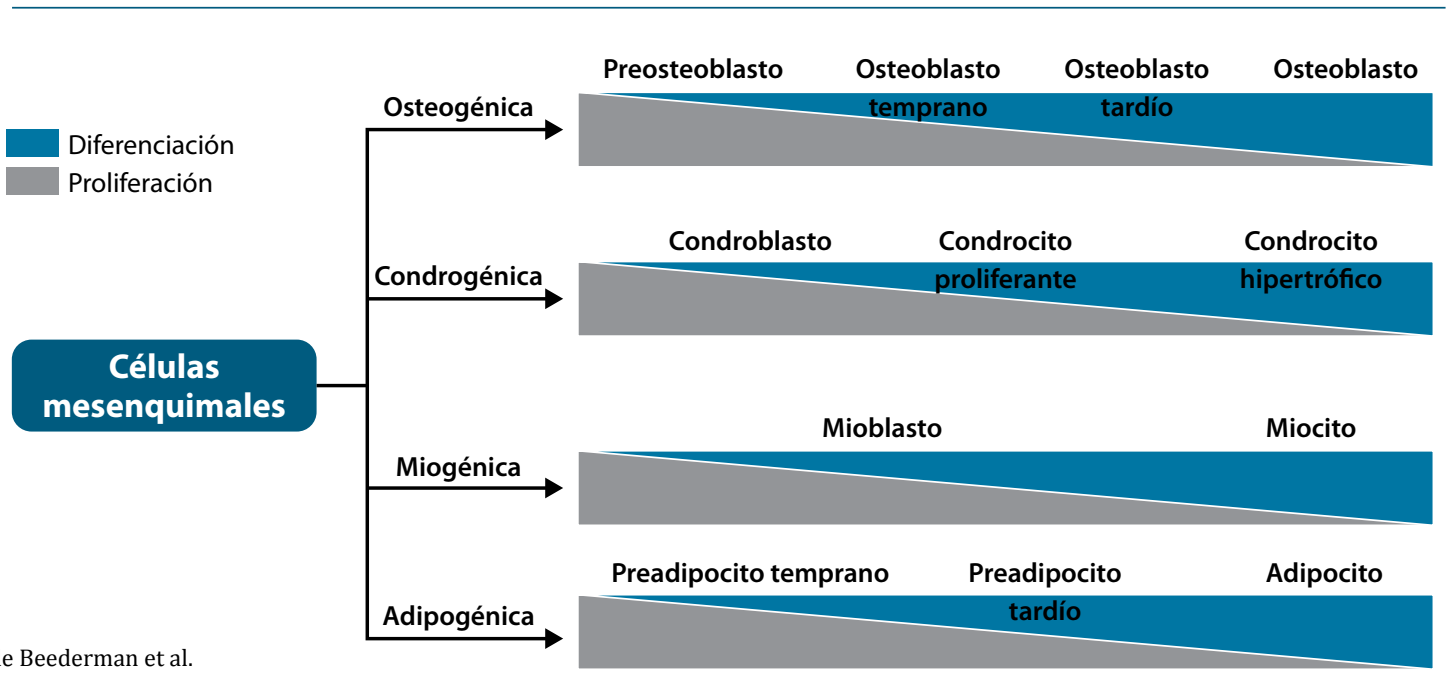


Figura 2. Factores de transcripción y moléculas de señalización asociados al desarrollo del osteoblasto y adipocito

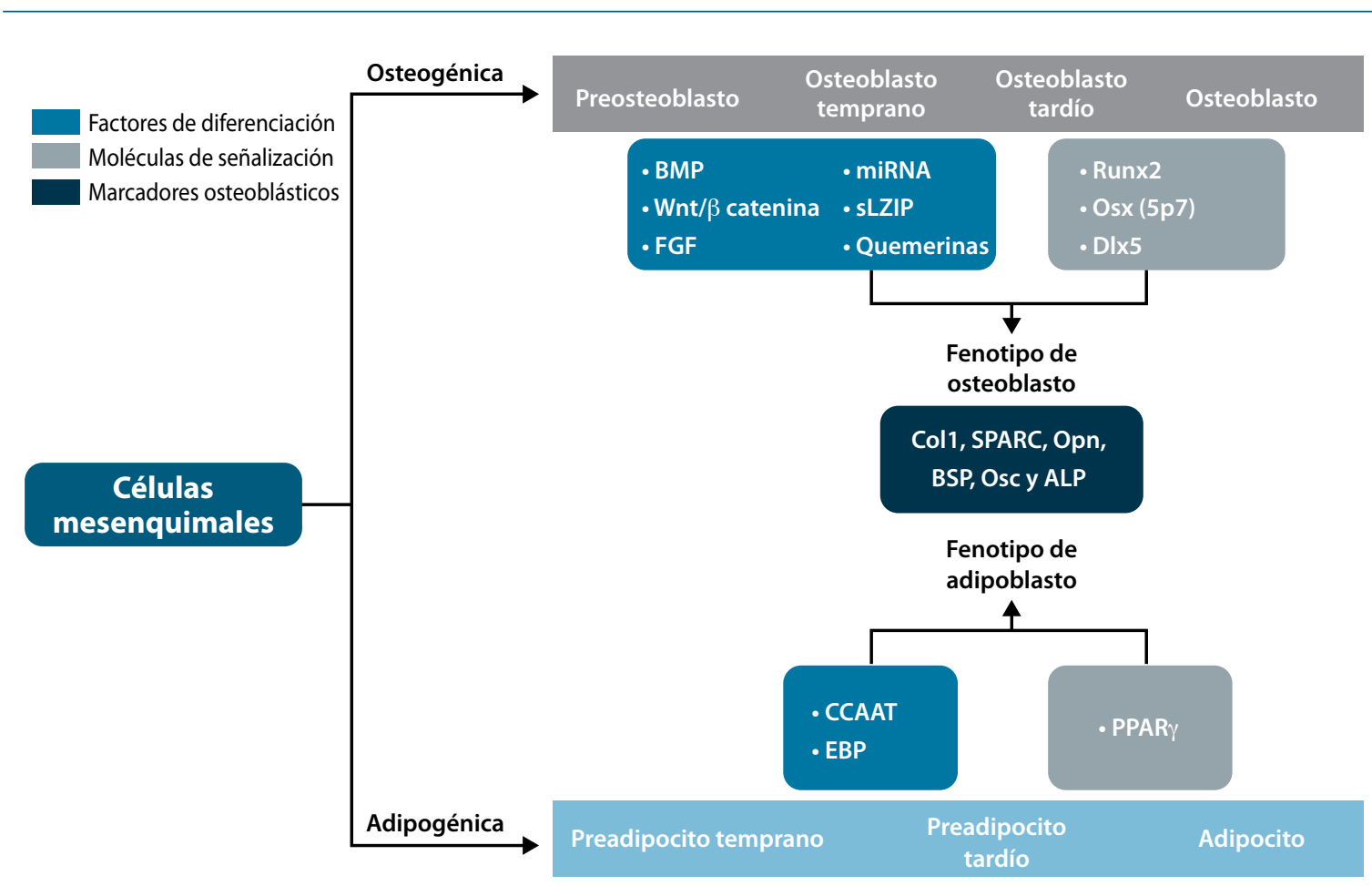

treonina (BMPR I y II), haciendo que el receptor activado por cinasas fosforile los factores de transcripción Smads 1, 5 y 8, los cuales, a nivel nuclear activan la expresión de genes objetivo con capacidad osteogénica y adipogénica ${ }^{(16)}$.

Mediante estudios de reacción en cadena de polimerasa cuantitativa (PCRc) en MSC se ha observado que la mayoría de las BMPs, con excepción de los 3 y 8, aumentan la regulación de la expresión de Runx2; sin embargo, solo los 2, 4, 6, 7 y 9 inducen formación de novo de hueso, lo cual se ha confirmado por la expresión de marcadores osteogénicos como la ALP. En modelos animales, se trasplantó subcutáneamente MSC con BMP 2 y 9; después de 5 semanas se observó, por imágenes de tomografía, proliferación y repoblación de masas sólidas, confirmando su importancia en la diferenciación linaje específica y en la osteogénesis, siendo BMP2 predominante para la osificación endocondral y BMP9 para la osificación intramembranosa $^{(16)}$.

\section{Wnt $/ \beta$ catenina}

La señalización inicia cuando las proteínas ligando se unen a receptores de superficie celular, llevando a la fosforilación de proteínas relacionadas con receptores LDL (LPR 5/6), con estabilización citoplasmática y aumento de la traslocación nuclear de $\beta$ catenina, lo que promueve la activación del factor de células $\mathrm{T}$ y el factor estimulador linfoide, que llevan a la activación de sus genes objetivo.
En estadios tempranos promueven la osteoblastogénesis y en estadios tardíos inhiben la maduración de osteoblastos diferenciados ${ }^{(17)}$. Para confirmar su influencia, fueron utilizados modelos animales con líneas transgénicas de pez cebra, que tienen características de osificación similar a los mamíferos. Se observó que la supresión de Wnt disminuye la osificación a nivel del opérculo, la mandíbula y los dientes, mientras que un aumento de su expresión se asoció de forma proporcional a aumento en la osificación, proceso mediado por activación de la Osx, por PCRc, se observaron cambios en la expresión de ésta, en hasta el $40 \%$ en casos control vs. en presencia de un inhibidor para Wnt. Así mismo se describió aumento de marcadores tempranos y tardíos de osteoblastogénesis tipo Wif1, Axin 2 y ALP en presencia de estimuladores para Wnt y disminución en presencia de inhibidores para la misma ${ }^{(18)}$.

En cultivos de MSC en medio adipoinductivo, con activación de PPAR $\gamma$ por medio de tiazolidinedionas, se observó una reducción del fenotipo osteoblastogénico evidenciado por disminución en la expresión de ALP, en relación a una naturaleza competitiva de las señales adipogénicas.

\section{FGF}

Con 23 miembros en 6 subfamilias, controlan la proliferación, diferenciación y supervivencia celular en varios tejidos, incluyendo el hueso. Tiene una acción altamente dependiente de la afinidad a los receptores, que genera activación de 
la fosfolipasa $\mathrm{C} \gamma$ (PLC $\gamma$ ), las proteínas cinasas activadas por mitógeno (MAPK) y la fosfatidilinositol 3 cinasa (PI3K), que finalmente se asocia a aumento de la expresión de genes osteoblásticos ${ }^{(19)}$.

En MSC, en condiciones basales hay una mayor expresión de los receptores para el factor de crecimiento fibroblástico (FGFR) tipo 1 y 2, el segundo que se asocia a diferenciación osteoblástica, ha mostrado ser un regulador positivo del crecimiento óseo y su supresión se asocia a dismorfismos óseos y disminución de la mineralización ósea ${ }^{(20)}$. En análisis de PCRc se observó aumento de 3-4 veces de la expresión de ARNm para Runx2, así mismo en cultivos de MSC mutados para FGFR2 hubo un aumento en los niveles de Osx, ALP y OC, en éstos a su vez se observó una mayor y más rápida calcificación de la matriz extracelular luego de 21 días y aumento de la expresión de SOST y DMP1 que son marcadores específicos para osteocitos, lo que indica efectividad en la promoción de la diferenciación osteoblástica temprana y terminal ${ }^{(21)}$, mientras que hubo una disminución de la expresión de factores de transcripción y de diferenciación adipocitaria como PPAR $\gamma$, EBP y LPL, evidenciando una diferenciación competitiva.

\section{sLZIP}

Los receptores nucleares requieren de la unión a coactivadores para lograr la transcripción de sus genes objetivo. Estos receptores mediante reguladores ligando dirigen el reclutamiento de los coactivadores por la unión entre el ligando y el receptor, induciendo un cambio de conformación que permite el reconocimiento de un motivo específico, el cual se conoce como caja NR o dominio LXXLL. A esta familia pertenecen los LZIP que se diferencian del resto de su familia por la ausencia del dominio transmembrana ${ }^{(22)}$.

En estudios in vitro e in vivo con cuantificación de la actividad de la luciferasa se observó en presencia de SLZIP inhibición de factores de transcripción adipogénica como el PPAR $\gamma 2$ hasta en un $32 \%$ y de la expresión de sus genes objetivo, como el FABP4 en un 68\%, C/EBP $\alpha$ en $42 \%$ y LPL en $76 \%$, con un aumento de la expresión del factor osteoblastogénico Runx2 en 4,2 veces y de los marcadores de diferenciación tardía tipo ALP, OC y BSP, muestra de la inhibición de la diferenciación adipocitaria y promoción de la osteoblastogénesis, mientras, que en presencia de ARN de interferencia para sLZIP, había un aumento de la actividad transcripcional de forma dosisdependiente de los factores adipogénicos ${ }^{(23,24)}$, ratificando nuevamente la diferenciación competitiva entre las líneas celulares derivadas de MSC.

En modelos animales, a nivel del proceso de formación ósea, se observó aumento de la DMO (36\%), volumen óseo (25\%), engrosamiento trabecular (22\%), número trabecular (31\%) y disminución de la separación trabecular en presencia de aumento de la expresión de $\operatorname{sLZIP}^{(23)}$.

\section{Quemerinas/CMKLR1}

Son adipocinas que regulan el crecimiento y metabolismo del adipocito, utilizan vías de señalización celular como el Notch, los ligandos logran activación de receptores transmembranales que resultan en la liberación de sus dominios intracelulares (NICD), éstos migran a su vez al núcleo para activar factores de transcripción que inducen la recombinación de señales de unión RBP-J con activación de transcripción de genes objetivos. Si hay una sobreexpresión constante se presenta inhibición de la osteoblastogénesis, mientras si es transitoria se logra estimulación de la diferenciación osteoblástica, mineralización y formación de hueso ectópico ${ }^{(25)}$.

El CMKLR1 aumenta en respuesta a estimulación con Wnt3a, ya conocido estimulador de osteoblastogénesis, de igual forma, se ha documentado una disminución de su expresión en presencia de PPARr y sus estimuladores. CMKLR1 regula la osteogénesis en MSC a través de una vía de ubiquitinización mediada por Wnt/ $\beta$ catenina. En cultivos celulares de MSC se observó que se requiere de Notch para mantener el balance entre los programas adipogénicos y osteoblastogénicos. En presencia de un bloqueo homocigoto para CMKLR1 hay pérdida de la diferenciación osteogénica $>70 \%$, con un aumento adipogénico proporcional, esto ratificado por resultados en ensayos immunoblot donde los marcadores osteogénicos tardíos como Oc, Col1A1, BSP y Osp disminuyen en la misma proporción.

\section{MiARN}

Descritos antes del año 2000, son pequeños fragmentos de ARN, no codificantes, que principalmente regulan la expresión génica de forma postranscripcional para el control de un amplio espectro de procesos biológicos, incluyendo autorrenovación, diferenciación, crecimiento y metabolismo de células madre ${ }^{(26)}$.

En el caso del miR-223 se ha observado regulación de la diferenciación de células estromales y MSC a adipocitos y osteoblastos. Por PCRc en tiempo real se observó un aumento de hasta 3 veces luego de $72 \mathrm{~h}$ de exposición a medio adipogénico, con aumento de niveles de factores de transcripción como PPAR $\gamma$ y EBP, así como de marcadores de diferenciación tipo aP2 y adipsina. En cultivos celulares hubo un aumento del $38 \%$ de adipocitos diferenciados, mientras que en medio osteogénico hubo una disminución del $40 \%$ de su expresión, con atenuación en los niveles de factores tipo Runx2 y de marcadores de diferenciación como APL, OC, Osp y FGFR2 ${ }^{(27)}$.

El miR-30 comprende una familia que incluye miR-30a, 30b, 30c, 30d y 30e, comparten una secuencia semilla y están asociados a diferenciación, senescencia y apoptosis celular. Regulan negativamente la diferenciación osteoblástica inducida por BMP2 a través de Smad1 y contribuyen a la formación adipocitaria. Por estudios de PCR en tiempo real se ha 
observado que el miR-30a y miR-30b aumentan su expresión en medio adipogénico sin cambios en medio osteogénico, el miR-30e aumenta en preadipocitos y el miR-30c y miR-30d aumentan en diferenciación adipocitaria y disminuyen durante la diferenciación osteoblástica, con el consecuente aumento de la expresión de factores de trascripción tipo PPAR $\gamma$, EBP y marcadores tipo aP2, con disminución de Runx2, Osx y ALP(28).

\section{Conclusión}

La homeostasis ósea depende de un eficaz pero delicado equilibrio entre la formación y la resorción ósea, mediado por la unidad funcional compuesta por los distintos grupos celulares que allí se encuentran. Estas células tienen un origen común a partir de las células multipontenciales mesenquimales, la presencia de disrupciones en el balance durante la diferenciación celular lleva a la presentación de fragilidad ósea y OP, se ha observado en estudios in vivo e in vitro que mutaciones en los factores de transcripción se asocian a defectos esqueléticos en consistencia con su rol en la diferenciación y función osteoblástica con un aumento proporcional por diferenciación competitiva de los adipocitos.

Análisis por microensayos han mostrado que las MSC de pacientes con OP primaria tienen un transcriptoma diferente en comparación de los controles sin OP y los donadores de MSC $\sin \mathrm{OP}^{(29)}$. Es por esto que debemos entender cuáles son y cómo funcionan las moléculas de señalización y los factores de transcripción que dirigen la expresión de estas células, puesto que son el futuro del tratamiento de distintas patologías óseas por medio de desdiferenciación y reprogramación celular. En la actualidad, ya se están desarrollando nuevas terapias basadas en la promoción de la regeneración ósea para OP primaria y secundaria ${ }^{(30,31)}$, así como el trasplante de MSC en la formación y regeneración ósea, en pacientes con traumas severos $^{(32)}$.

\section{Declaración de fuentes de financiación y posibles conflic- tos de interés \\ Ninguno}

\section{Referencias}

1. Curtis EM, Moon RJ, Dennison EM, Harvey NC, Cooper C. Recent advances in the pathogenesis and treatment of osteoporosis. Clin Med. 2016;16(4):360364.

2. Paula FJADE, Black DM, Rosen CJ. CAPÍTULO 29 Osteoporosis y biología ósea. 13th Editi. Williams. Tratado de endocrinología. Elsevier España, S.L.U.; 2017. 1323-1364 p.

3. Armas LAG, Recker RR. Pathophysiology of Osteoporosis. New Mechanistic Insights. Endocrinol Metab Clin North Am. 2012;41(3):475-86.

4. Rosen CJ, Ackert-Bicknell C, Rodriguez JP, Pino AM. Marrow fat and the bone microenvironment: developmental, functional, and pathological implications. Crit Rev Eukaryot Gene Expr. 2009;19(2):109-24.

5. Feng X, McDonald JM. Disorders of Bone Remodeling. Annu Rev Pathol Mech Dis. 2011;6(1):121-45.

6. Eriksen EF. Cellular mechanisms of bone remodeling. Rev Endocr Metab Disord. 2010;11(December):219-27.

7. Zayzafoon M, Gathings WE, McDonald JM. Modeled microgravity inhibits osteogenic differentiation of human mesenchymal stem cells and increases adipogénesis. Endocrinology. 2004;145(5):2421-32.

8. Rubin MR. Skeletal fragility in diabetes. Ann N Y Acad Sci. 2017;1402(1):1830.

9. Güler-Yüksel M, Hoes JN, Bultink IEM, Lems WF. Glucocorticoids, Inflammation and Bone. Calcif Tissue Int. 2018;7.

10. Beederman M, Lamplot JD, Nan G, Wang J, Liu X, Yin L, et al. HHS Public Access. 2016;6:32-52.

11. Chamberlain G, Fox J, Ashton B, Middleton J. Concise Review: Mesenchymal Stem Cells: Their Phenotype, Differentiation Capacity, Immunological Features, and Potential for Homing. Stem Cells. 2007;25(11):2739-49.

12. Berendsen $\mathrm{AD}$, Olsen $\mathrm{BR}$. Osteoblast-adipocyte lineage plasticity in tissue development, maintenance and pathology. Cell Mol Life Sci. 2014;71(3):493-7.

13. Kawai M. Adipose tissue and bone: Role of PPAR?? in adipogénesis and osteogenesis. Horm Mol Biol Clin Investig. 2013;15(3):105-13.

14. Kawai M, Rosen CJ. PPAR??: A circadian transcription factor in adipogénesis and osteogenesis. Vol. 6, Nature Reviews Endocrinology. 2010. p. 629-36.

15. Yu WH, Li FG, Chen XY, Li JT, Wu YH, Huang LH, et al. PPAR $\gamma$ suppression inhibits adipogénesis but does not promote osteogenesis of human mesenchymal stem cells. Int J Biochem Cell Biol. 2012;44(2):377-84.

16. Kang $\mathrm{Q}$ Song W-X, Luo $\mathrm{Q}$, Tang N, Luo J, Luo X, et al. A Comprehensive Analysis of the Dual Roles of BMPs in Regulating Adipogenic and Osteogenic Differentiation of Mesenchymal Progenitor Cells. Stem Cells Dev. 2009;18(4):545-58

17. Lerner UH, Ohlsson C. The WNT system: Background and its role in bone. Intern Med. 2015;277(6):630-49.

18. Felber K, Elks PM, Lecca M, Roehl HH. Expression of osterix is regulated by FGF and Wnt/ $\beta$-catenin signalling during osteoblast differentiation. PLoS One. 2015;10(12):1-17.
19. Kähkönen TE, Ivaska KK, Jian M, Büki KG, Väänänen HK, Härkönen PL. Role of fibroblast growth factor receptors (FGFR) and FGFR like-1 (FGFRL1) in mesenchymal stromal cell differentiation to osteoblasts and adipocytes. Mol Cell Endocrinol. 2017;1.

20. Du X, Xie Y, Xian CJ, Chen L. Role of FGFs/FGFRs in skeletal development and bone regeneration. J Cell Physiol. 2012;227(12):3731-43.

21. Miraoui H, Oudina K, Petite H, Tanimoto Y, Moriyama K, Marie PJ. Fibroblast growth factor receptor 2 promotes osteogenic differentiation in mesenchymal cells via ERK1/2 and protein kinase C signaling. J Biol Chem. 2009;284(8):4897-904.

22. Chadwick AC, Jensen DR, Hanson PJ, Lange PT, Proudfoot SC, Peterson FC et al. NMR Structure of the C-Terminal Transmembrane Domain of the HDL Receptor, SR-BI, and a Functionally Relevant Leucine Zipper Motif. Structure. 2017;25(3):446-57.

23. Kim J, Ko J. A novel PPAR $\gamma 2$ modulator sLZIP controls the balance between adipogénesis and osteogenesis during mesenchymal stem cell differentiation. Cell Death Differ. 2014;21(10):1642-55.

24. Pan G, Cao J, Yang N, Ding K, Fan C, Xiong WC, et al. Role of glucocorticoid-induced leucine zipper (GILZ) in bone acquisition. J Biol Chem. 2014;289(28):19373-82.

25. Scotia N, Chemistry P, Building R, Uni- TOS, Universi- D, Scotia N, et al. Chemokine-Like Receptor 1 ( CMKLR1) is a Novel Wnt Target Gene that Regulates Mesen- chymal Stem Cell Differentiation. 2016;

26. Zeng ZL, Lin X long, Tan LL, Liu YM, Qu K, Wang Z. MicroRNAs: Important Regulators of Induced Pluripotent Stem Cell Generation and Differentiation. Stem Cell Rev Reports. 2017;0(0):1-11.

27. Guan X, Gao Y, Zhou J, Wang J, Zheng F, Guo F, et al. MiR-223 regulates adipogenic and osteogenic differentiation of mesenchymal stem cells through a C/EBPs/miR-223/FGFR2 regulatory feedback loop. Stem Cells. 2015;33(5):1589-600.

28. Wang J, Guan X, Guo F, Zhou J, Chang A, Sun B, et al. miR-30e reciprocally regulates the differentiation of adipocytes and osteoblasts by directly targeting low-density lipoprotein receptor-related protein 6. 2013;

29. Phetfong J, Sanvoranart T, Nartprayut K, Nimsanor N, Seenprachawong K, Prachayasittikul V, et al. Osteoporosis: the current status of mesenchymal stem cell-based therapy. Cell Mol Biol Lett. 2016;21(1):12.

30. Pino AM, Rosen CJ, Pablo Rodríguez J. In Osteoporosis, differentiation of mesenchymal stem cells (MSCs) improves bone marrow adipogénesis. Biol Res. 2012;45(3):279-87.

31. Rocha MA de C, Silva LMC, Oliveira WA de, Bezerra D de O, Silva GC da, Silva $\mathrm{L}$ dos $\mathrm{S}$, et al. Allogeneic mesenchymal stem cells and xenogenic platelet rich plasma, associated or not, in the repair of bone failures in rabbits with secondary osteoporosis. Acta Cir Bras. 2017;32(9):767-80.

32. Burdette AJ, Guda T, Thompson ME, Banas R, Sheppard F. A Novel Secretome Biotherapeutic Influences Regeneration in Critical Size Bone Defects. J Craniofac Surg. 2017;0(0):1. 